CLINICAL STUDY

\title{
Skeletal muscle mitochondrial function in polycystic ovarian syndrome
}

\author{
Rasmus Rabøl $^{1,3, *}$, Pernille F Svendsen ${ }^{2, *}$, Mette Skovbro ${ }^{1}$, Robert Boushel ${ }^{1}$, Peter Schjerling ${ }^{1,4}$, Lisbeth Nilas $^{2}$, \\ Sten Madsbad ${ }^{3}$ and Flemming Dela ${ }^{1}$ \\ ${ }^{1}$ Department of Biomedical Sciences, Faculty of Health Sciences, Center for Healthy Aging, University of Copenhagen, Blegdamsvej 3b, DK-2200 \\ Copenhagen N, Denmark, Departments of ${ }^{2}$ Gynecology and Obstetrics and ${ }^{3}$ Endocrinology, Hvidovre University Hospital, University of Copenhagen, \\ Hvidovre, Denmark and ${ }^{4}$ Institute of Sports Medicine Copenhagen, Bispebjerg Hospital, Copenhagen, Denmark \\ (Correspondence should be addressed to R Rabøl at Department of Biomedical Sciences, University of Copenhagen; Email: rasmus.rabol@dadlnet.dk) \\ *(R Rabøl and P F Svendsen contributed equally to this work)
}

\begin{abstract}
Objective: Polycystic ovarian syndrome (PCOS) is associated with skeletal muscle insulin resistance (IR), which has been linked to decreased mitochondrial function. We measured mitochondrial respiration in lean and obese women with and without PCOS using high-resolution respirometry.

Methods: Hyperinsulinemic-euglycemic clamps $\left(40 \mathrm{mU} / \mathrm{min}\right.$ per $\mathrm{m}^{2}$ ) and muscle biopsies were performed on 23 women with PCOS (nine lean (body mass index (BMI) $<25 \mathrm{~kg} / \mathrm{m}^{2}$ ) and 14 obese $\left(\mathrm{BMI}>25 \mathrm{~kg} / \mathrm{m}^{2}\right)$ ) and 17 age- and weight-matched controls (six lean and 11 obese). Western blotting and high-resolution respirometry was used to determine mitochondrial function.

Results: Insulin sensitivity decreased with PCOS and increasing body weight. Mitochondrial respiration with substrates for complex I and complex I+ II were similar in all groups, and PCOS was not associated with a decrease in mitochondrial content as measured by mitochondrial DNA/genomic DNA. We found no correlation between mitochondrial function and indices of insulin sensitivity. Conclusions: In contrast to previous reports, we found no evidence that skeletal muscle mitochondrial respiration is reduced in skeletal muscle of women with PCOS compared with control subjects. Furthermore, mitochondrial content did not differ between our control and PCOS groups. These results question the causal relationship between reduced mitochondrial function and skeletal muscle IR in PCOS.
\end{abstract}

European Journal of Endocrinology 165 631-637

\section{Introduction}

Polycystic ovarian syndrome (PCOS) is characterized by chronic anovulation, hyperandrogenism, and polycystic ovaries (1). It is the most common endocrinopathy in younger women, with an estimated prevalence of $5-$ $10 \%$ in the general population (2). Insulin resistance (IR) is considered the main pathological factor responsible for the hormonal disturbances of the syndrome (3), and IR has been associated with reduced mitochondrial respiration (4). In patients with PCOS, reduced expression of nuclear-encoded genes involved in oxidative phosphorylation (OXPHOS) has been demonstrated in skeletal muscle (5). This reduction in expression of OXPHOS genes is similar to what has been demonstrated in patients with type 2 diabetes (6) and subjects with a family history of type 2 diabetes (7).

It is still debated whether a possible reduction in OXPHOS gene expression is reflected in reduced overall function of skeletal muscle mitochondria. Studies of oxygen consumption in blood mononuclear cells (leukocytes) have indicated that mitochondrial complex I respiration is reduced in women with PCOS compared with age- and body mass index (BMI)-matched control subjects (8). However, the causal link between leukocyte mitochondrial impairment and muscular IR remains elusive. It has been speculated that leukocyte mitochondrial impairment leads to increased reactive oxygen species production and increased production of proinflammatory markers such as TNF $\alpha$ causing IR (8). The demonstration of impaired skeletal muscle mitochondrial function would provide stronger evidence for the central role of mitochondrial impairment in PCOS, as skeletal muscle is the primary site for IR. Thus, we wanted to test the hypothesis that skeletal muscle mitochondrial respiratory capacity is reduced in PCOS, linking mitochondrial impairment to the muscular IR that is central to the pathophysiology of PCOS. Therefore, we applied the method of high-resolution respirometry (9) on permeabilized skeletal muscle fibers 
in women with PCOS to thoroughly characterize mitochondrial respiration. High-resolution respirometry allows for detailed measurements of electron transport chain function through successive addition of substrates and inhibitors. We compared mitochondrial function in groups of normal weight and obese women with PCOS to age- and BMI-matched control groups to distinguish between the effects of PCOS and the effects of obesity on mitochondrial function.

\section{Methods}

\section{Subjects}

A total of 40 women were included in the study. All were recruited by advertising in the local newspaper. The participants were screened by blood sample analyses for testosterone and DHAS, and other endocrinopathies were excluded by relevant testing. Polycystic ovarian morphology was determined by transvaginal ultrasonography and categorized in accordance with the Rotterdam criteria. The patients and control subjects in this study are subgroups from a larger cohort $(10,11)$.

Of the 40 women, 23 women had PCOS, nine lean (lean PCOS, BMI $<25 \mathrm{~kg} / \mathrm{m}^{2}$ ), and 14 obese (obese PCOS, BMI $>25 \mathrm{~kg} / \mathrm{m}^{2}$ ). In the group of lean PCOS subjects, all subjects had polycystic ovaries on ultrasonography, seven subjects had oligo or amenorrhea, four subjects had clinical hyperandrogenism, and seven subjects had hyperandrogenemia. In the group of obese PCOS subjects, all subjects had polycystic ovaries on ultrasonography, eight subjects had oligo or amenorrhea, seven subjects had clinical hyperandrogenism, and 11 subjects had hyperandrogenemia.

Since the majority of women with PCOS were oligo or amenorrhoeic, blood samples and tests were therefore taken at random both in women with and without PCOS.

Seventeen age- and weight-matched control women, six lean (lean controls, BMI $<25 \mathrm{~kg} / \mathrm{m}^{2}$ ) and 11 obese (obese controls, BMI $>25 \mathrm{~kg} / \mathrm{m}^{2}$ ), were identified. All women in the control group had regular menstrual cycles $(<35$ days) and had androgen levels within the normal range. One subject in the group of lean control subjects and eight subjects in the group of obese controls had polycystic ovaries on ultrasonography but none had clinical hyperandrogenism.

Body composition was estimated by a whole-body dual energy X-ray absorptiometry scan (Hologic QDR2000, Waltham, MA, USA). Women with other known chronic diseases and women who had used oral contraceptives or other drugs known to alter glucose and insulin metabolism within the last 3 months, were not included in the study. The study was conducted according to the principles expressed in the 'Declaration of Helsinki', and approved by the local ethics committee. All subjects gave written informed consent before entering the study.

\section{Oral glucose tolerance test}

After a $10 \mathrm{~h}$ overnight fast a standard oral glucose tolerance test $(75 \mathrm{~g})$ was performed. Blood samples were drawn from the antecubital vein at 0 and $120 \mathrm{~min}$ for measurement of plasma glucose concentrations. Patients with a $2 \mathrm{~h}$ plasma glucose concentration $<7.8 \mathrm{mmol} / \mathrm{l}$ were categorized as having normal glucose tolerance, $>7.8$ and $<11.1 \mathrm{mmol} / \mathrm{l}$ impaired glucose tolerance, and $>11.1 \mathrm{mmol} / \mathrm{l}$ diabetes mellitus.

The baseline glucose values were similar between the groups (Fig. 1), but the $2 \mathrm{~h}$ value was higher in the obese groups compared with the lean controls (lean PCOS versus obese PCOS: $6.0+0.4$ vs $7.1+0.3 \mathrm{mmol} / \mathrm{l}$, $P<0.05$ ) (lean control versus obese control: $4.9+0.5$ vs $7.0+0.3 \mathrm{mmol} / \mathrm{l}, P<0.01)$.

\section{Euglycemic-hyperinsulinemic clamp}

After an overnight fast of 10-12 h all women reported to the laboratory between 0800 and $0830 \mathrm{~h}$. Instructions were given to abstain from any strenuous physical activity 3 days before the assessment. A catheter was inserted in the right and left antecubital veins. The right catheter was used for blood sampling and the left for later infusion of insulin and glucose. The right arm was kept in a heating box to obtain arterialized venous blood. After a $30 \mathrm{~min}$ rest, insulin (Actrapid; Novo Nordisk A/S, Bagsværd, Denmark) was infused at a rate of $100 \mathrm{mU} / \mathrm{min}$ per $\mathrm{m}^{2}$ followed by a stepwise decline in infusion rate every third minute by $20 \mathrm{mU} / \mathrm{min}$ per $\mathrm{m}^{2}$, until an infusion rate of $40 \mathrm{mU} / \mathrm{min}$ per $\mathrm{m}^{2}$ was reached after $9 \mathrm{~min}$. This rate was maintained for $120 \mathrm{~min}$. Plasma glucose concentrations were measured every $5 \mathrm{~min}$, and kept at $5 \mathrm{mmol} / \mathrm{l}$ by adjusting the glucose $(200 \mathrm{~g} / \mathrm{l})$ infusion rate. Blood samples for assessing plasma insulin were taken at $10,30,60,90$, and $120 \mathrm{~min}$. Insulin concentrations during steady state did not differ between the groups (lean PCOS, 462 + $32 \mathrm{pmol} / \mathrm{l}$; obese PCOS, $464+27 \mathrm{pmol} / \mathrm{l}$; lean control,
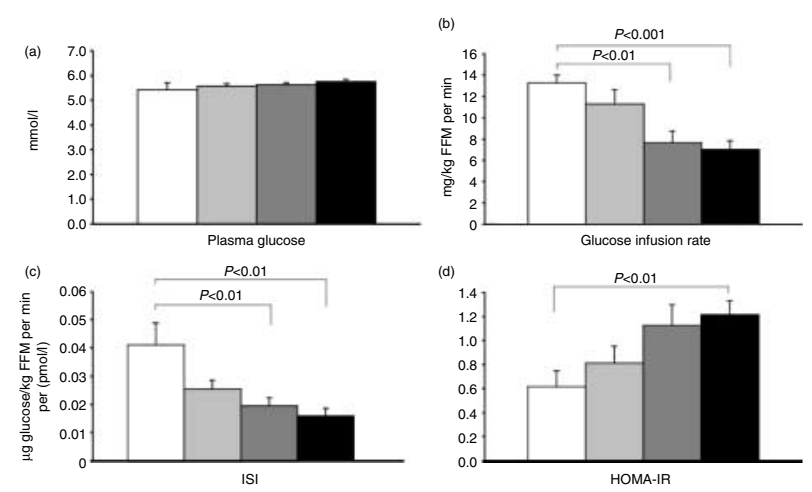

Figure 1 Basal plasma glucose levels (a), glucose infusion rates (b), ISI values (c), and HOMA-IR values (d) for the four groups of subjects. Data are shown as mean \pm S.E.M. White: lean control $(n=6)$. Light gray: lean PCOS $(n=9)$. Dark gray: obese controls $(n=11)$. Black: obese PCOS $(n=14)$. 
$362+51 \mathrm{pmol} / \mathrm{l}$; obese control, $419+27 \mathrm{pmol} / \mathrm{l})$, and steady-state plasma insulin concentrations were achieved between 10 and $30 \mathrm{~min}$ (data not shown).

Plasma glucose was measured by a Beckman glucose analyzer (Ramcon, Fullerton, CA, USA).

\section{Laboratory analyses}

Testosterone was measured by RIA after ether extraction and subsequent celite chromatography. The intra- and inter-assay variations were 8.2 and $13.8 \%$ respectively. Free testosterone was estimated as described previously (10).

Blood samples for analyzing insulin were centrifuged immediately at $4{ }^{\circ} \mathrm{C}$, and plasma was stored at $-80{ }^{\circ} \mathrm{C}$ for later analysis. Insulin was analyzed by 1235 Auto DELPHIA automatic immunoassay system (Wallac, Oy, Turku, Finland), with a detection limit of $3 \mathrm{pmol} / \mathrm{l}$. The intraand inter-assay coefficients of variance were 4.5 and $7 \%$.

\section{Calculations}

Glucose disposal rate was determined during the final steady-state period of the clamp ( $t=90-120 \mathrm{~min})$, and calculated as milligrams per kilogram fat-free mass per minute. The insulin sensitivity index (ISI) was calculated as (glucose disposal rate)/(plasma insulin during last $30 \mathrm{~min}$ of clamp), expressed in micrograms of glucose per $\mathrm{kg}$ fat-free mass per minute/(pmol/l). The homeostasis model assessment IR index (HOMA-IR) was used for the evaluation of whole-body insulin sensitivity. The HOMA-IR values were calculated using the HOMACalculator, version 2.2 software (Diabetes Trials Unit, University of Oxford, Oxford, UK).

\section{Muscle biopsies}

Preparation of muscle fibers Muscle biopsies were taken from $\mathrm{m}$. vastus lateralis using the Bergstrøm needle technique. Muscle biopsies were taken at baseline before the clamp was performed. A small part of the biopsy sample $(5 \mathrm{mg})$ was immediately placed in ice-cold relaxing (BIOPS) solution and treated with saponin $(50 \mu \mathrm{g} / \mathrm{ml})$ as described previously (12). Measurements of oxygen consumption were performed at $37{ }^{\circ} \mathrm{C}$ using a high-resolution respirometer (Oroboros Instruments, Innsbruck, Austria), see below. The rest of the biopsy samples were frozen in liquid nitrogen and stored at $-80{ }^{\circ} \mathrm{C}$ for later analysis.

Mitochondrial respiration protocol To avoid any potential oxygen diffusion limitation all experiments were conducted after hyper-oxygenation $(\sim 450 \mathrm{nmol}$ $\mathrm{O}_{2} / \mathrm{ml}$ ). Substrates and inhibitors were added consecutively. State 3 respiration with electron flux through complex I (state GM3) was measured after the addition of malate $(2 \mathrm{mmol} / \mathrm{l})$, glutamate $(10 \mathrm{mmol} / \mathrm{l})$, and ADP $(5 \mathrm{mmol} / \mathrm{l})$, and maximal coupled respiration with convergent electron flux through complex I and complex II (state GMS3), was achieved by adding succinate $(10 \mathrm{mmol} / \mathrm{l})$ to the muscle biopsy in the respiration chamber. The integrity of the outer mitochondrial membrane was subsequently assessed by the addition of cytochrome $c(10 \mathrm{mmol} / \mathrm{l})$ in state GMS3. In all studies, addition of cytochrome $c$ had no additive effect of more than $20 \%$ on respiration confirming the integrity of the outer mitochondrial membrane. We examined uncoupled respiration by addition of the protonophore carbonylcyanide-4-(trifluoromethoxy)phenylhydrazone (FCCP; $1 \mathrm{~mol} / \mathrm{l})$ in a stepwise titration. The addition of rotenone $(0.5 \mathrm{mmol} / \mathrm{l})$ resulted in inhibition of complex I for examination of $\mathrm{O}_{2}$ flux with complex II substrate alone. Tissue wet weight was used as the denominator for oxygen flux rates.

Mitochondrial DNA measurement For measurement of mitochondrial DNA (mtDNA) content, DNA was isolated from $\sim 10 \mathrm{mg}$ muscle biopsies by proteinase $\mathrm{K}$ digestion at $55^{\circ} \mathrm{C}$ for 3 days. The $100 \mu$ l digestion mix contained $50 \mathrm{mU}$ proteinase $\mathrm{K}$ (PCR grade, Roche), $20 \mathrm{mmol} / \mathrm{l}$ Tris-HCl (pH 8.4), and $50 \mathrm{mmol} / \mathrm{l} \mathrm{KCl}$. After incubation at $80{ }^{\circ} \mathrm{C}$ for $45 \mathrm{~min}$, the remains were spun down; and the supernatant was diluted 200 times in

Table 1 Baseline characteristics

\begin{tabular}{|c|c|c|c|c|c|c|}
\hline & \multicolumn{3}{|c|}{ Obese } & \multicolumn{3}{|c|}{ Lean } \\
\hline & $\begin{array}{l}\text { PCOS } \\
n=14\end{array}$ & $\begin{array}{c}\text { Control } \\
n=11\end{array}$ & $\begin{array}{c}\boldsymbol{P} \\
\text { PCOS vs control }\end{array}$ & $\begin{array}{c}\text { PCOS } \\
n=9\end{array}$ & $\begin{array}{c}\text { Control } \\
n=6\end{array}$ & $\begin{array}{c}\boldsymbol{P} \\
\text { PCOS vs control }\end{array}$ \\
\hline Age (years) & $29.6 \pm 1.2$ & $32.2 \pm 1.4$ & NS & $28.3 \pm 1.6$ & $30.7 \pm 1.6$ & NS \\
\hline Weight (kg) & $95.1 \pm 2.4$ & $92.6 \pm 3.5$ & NS & $64.7 \pm 2.3$ & $59.7 \pm 3.0$ & NS \\
\hline Height (cm) & $169.0 \pm 1.6$ & $165.9 \pm 1.2$ & NS & $166.6 \pm 2.4$ & $162.7 \pm 3.1$ & NS \\
\hline BMI $\left(\mathrm{kg} / \mathrm{m}^{2}\right)$ & $33.4 \pm 1.0$ & $33.6 \pm 1.2$ & NS & $23.3 \pm 0.7$ & $22.5 \pm 0.3$ & NS \\
\hline \multicolumn{7}{|l|}{ Plasma } \\
\hline Fasting insulin (pmol/l) & $62.6 \pm 6.1$ & $59.1 \pm 9.1$ & NS & $42.4 \pm 7.3$ & $30.7 \pm 7.7$ & NS \\
\hline $\mathrm{FA}(\mathrm{mmol} / \mathrm{l})$ & $0.46 \pm 0.05$ & $0.53 \pm 0.04$ & NS & $0.61 \pm 0.08$ & $0.53 \pm 0.19$ & NS \\
\hline $\mathrm{TG}(\mathrm{mmol} / \mathrm{l})$ & $0.95 \pm 0.08$ & $1.07 \pm 0.17$ & NS & $0.91 \pm 0.16$ & $0.87 \pm 0.07$ & NS \\
\hline \multicolumn{7}{|l|}{ Testosterone (nmol/l) } \\
\hline Free & $0.045 \pm 0.006$ & $0.024 \pm 0.002$ & $<0.01$ & $0.036 \pm 0.006$ & $0.013 \pm 0.002$ & $<0.05$ \\
\hline Total & $2.408 \pm 0.25$ & $1.455 \pm 0.11$ & $<0.01$ & $2.257 \pm 0.01$ & $1.405 \pm 0.16$ & NS \\
\hline
\end{tabular}



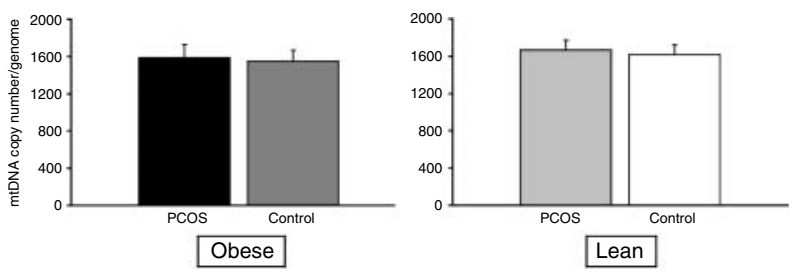

Figure 2 Skeletal muscle mitochondrial content measured as mtDNA copy number/genome. No statistical significant differences between the groups were found. Data are shown as mean \pm S.E.M.

Tris-EDTA plus $1 \mathrm{ng} / \mu \mathrm{l}$ Salmon Sperm DNA (Sigma). Five microliters of this dilution was amplified in a $25 \mu \mathrm{l}$ PCR containing $1 \times$ Quantitect SYBR Green Master Mix (Qiagen) and $100 \mathrm{nmol} / \mathrm{l}$ of each primer. The amplification was monitored real-time using the MX3000P Real-time PCR machine (Stratagene, La Jolla, CA, USA). The primers were designed to target genomic DNA (myogenin promoter: AGG TGC TGT CAG GAA GCA AGG A and TAG GGG GAG GAG GGA ACA AGG A) or mtDNA (COX1: CCC CTG CCATAACCCAATACC A and CCA GCA GCTAGG ACT GGG AGA GA). The $C_{\mathrm{t}}$ values were related to a standard curve made with the cloned PCR products. These PCR products were constructed by TA cloning of the real-time PCR products into pDrive (Qiagen PCR Cloning Kit, Qiagen) followed by PCR amplification of single clones with M13 primers (CCA GGG TTT TCC CAG TCA CGA and AGC GGATAA CAATTT CAC ACA GGA) and purification with a PCR purification kit (High Pure PCR purification kit, Roche). These PCR products were then sequence verified (insertion opposite direction of lacZ); and via quantification by spectrophotometry $(260 \mathrm{~nm})$, a dilution series (the standard curve) was made in TE plus $1 \mathrm{ng} / \mu \mathrm{l}$ Salmon Sperm DNA (Sigma) with known number of molecules $(5 \times 106$ down to 10 molecules per PCR $)$. mtDNA content per genome was then calculated as the ratio of the molecules measured with the mtDNA primers and the genomic DNA primers for each sample.

\section{Statistical analysis}

All comparisons were calculated by two-tailed Student's $t$-test. $P$ values $<0.05$ were considered significant. Tests for correlations were calculated using Pearson correlation coefficient $(r)$. All data are expressed as means + s.E.M.

\section{Results}

\section{Glucose homeostasis}

Groups were well-matched with regards to age and BMI (Table 1). Basal plasma glucose was similar between the groups (Fig. 1a), and plasma fasting insulin was not different between obese PCOS and obese controls and between lean PCOS and lean controls (Table 1). Fasting plasma insulin was higher in obese PCOS compared with lean PCOS $(62.6+6.1$ vs $42.4+7.3 \mathrm{pmol} / \mathrm{l}$, $P<0.05)$ and tended to be higher in the obese controls compared with lean controls $(59.1+9.1$ vs 30.7 $+7.7 \mathrm{pmol} / \mathrm{l}, P=0.06$ ). Glucose infusion rates during the steady-state period of the clamp were highest in the lean controls and were significantly lower in obese controls and obese PCOS (Fig. 1b). The ISI showed the same pattern with the lean controls being the most sensitive and the lean PCOS $(P=0.53$ versus lean controls), obese controls ( $P<0.01$ versus lean controls), and obese PCOS $(P<0.01$ versus lean controls) being progressively more insulin resistant (Fig. 1c). The HOMA-IR values calculated on the basis on fasting plasma glucose and insulin showed the same pattern as glucose infusion rates and ISI (Fig. 1d).

\section{Mitochondrial function}

Mitochondrial content measured as mtDNA/genomic DNA did not differ between the groups (Fig. 2).

Mitochondrial coupled respiration with substrates for complex I (state GM3: malate, glutamate, and ADP) and complex I+ II (state GMS3: + succinate) did not differ between PCOS and control in the obese or in the lean group. However, state GMS3 was significantly $(P<0.05)$ lower in the obese PCOS versus the lean PCOS (Fig. 3). Likewise, maximally uncoupled respiration, representing maximal electron flux capacity, was significantly $(P<0.05)$ lower in obese PCOS compared with obese controls (Fig. 4). Uncoupled respiration did not differ between control and PCOS in the lean groups. The uncoupling control ratio (UCR: the ratio between FCCP respiration and state GMS3 (9)) was significantly higher in the obese control $(1.7+0.1)$ than any of the other groups (obese PCOS, $1.3+0.1$; lean PCOS, 1.2 +0.1 ; lean controls, $1.2+0.1, P<0.01$ for all comparisons), implying that obese subjects have excess capacity for electron transport.

With the final addition of rotenone, inhibiting complex I respiration, the respiratory rates decreased similarly in the groups (obese PCOS versus obese controls: $-43+2$ vs $-40+2 \%$ ) or between the lean groups (lean PCOS versus lean control: $-39+3$ vs $38+6 \%)$ and the lower respiration in obese PCOS
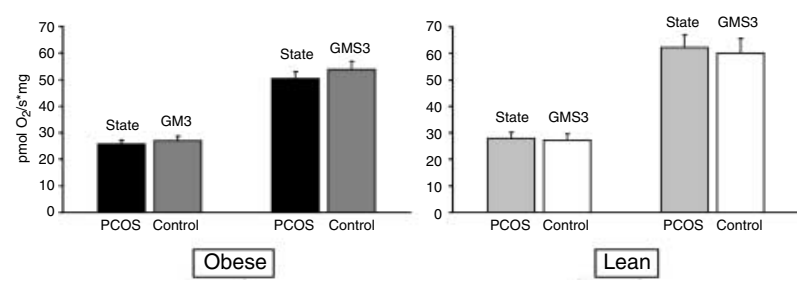

Figure 3 Coupled mitochondrial respiration using substrates for complex I (state GM3: malate, glutamate, and ADP) and complex I+ II (state GMS3: + succinate). No significant differences within the groups of obese subjects or within the groups of lean subjects were found. However, state GMS3 was significantly lower in the obese PCOS versus the lean PCOS. Data are shown as mean \pm S.E.M. 

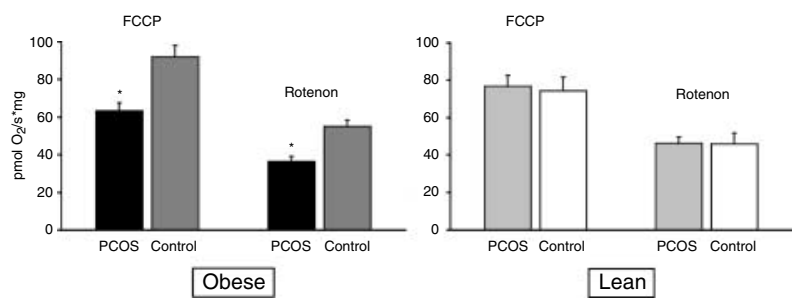

Figure 4 Uncoupled respiration after the addition of FCCP. Obese control subjects have significantly higher rates of respiration compared with obese PCOS. Respiration after complex I inhibition was also higher in the obese controls compared with obese PCOS. No differences were found between the lean groups. Data are shown as mean \pm S.E.M. ${ }^{*} P<0.001$.

versus obese controls was maintained. This demonstrates that complex I activity is not reduced in skeletal muscle of women with PCOS.

Mitochondrial respiration did not correlate with insulin sensitivity measured by hyperinsulinemic-euglycemic clamping (Fig. 5). GM3 respiration $\left(r^{2}=0.02, n=39\right.$, NS), GMS3 respiration $\left(r^{2}=0.1, n=39\right.$, NS), and FCCP respiration $\left(r^{2}=0.04, n=39\right.$, NS) were not significantly correlated with the glucose infusion rate. Significant correlations between these parameters were not found in any of the subgroups either.

\section{Discussion}

This study demonstrates that PCOS is not associated with deficiencies in coupled mitochondrial respiration in skeletal muscle in either obese or lean subjects, and that PCOS is not associated with any deficiencies in complex I activity. Thus, OXPHOS capacity is intact in PCOS, and the previously reported downregulation of genes involved in OXPHOS (5) in women with PCOS and IR is not translated into reduced overall function of coupled mitochondrial respiration, and the reported reductions in complex I activity in leukocytes (8) is not found in skeletal muscle.

Mitochondrial respiration in skeletal muscle biopsies was measured by high-resolution respirometry. The measurements are a composite of citric acid cycle flux and electron transport chain activity and provide a good marker of overall mitochondrial function. Our subjects were well-matched on age and BMI and the design of the study allows us to draw conclusions regarding the effect of PCOS and the effect of obesity on mitochondrial function. The rather small difference in insulin sensitivity between the PCOS group and control subjects might be explained by the fact that not all PCOS subjects displayed hyperandrogenism despite menstrual dysfunction and polycystic ovaries on ultrasonography.

As in several of our previous studies, we did not find any significant correlation between mitochondrial function and measures of insulin sensitivity (12-16) challenging the concept of a direct causal relationship between mitochondrial function and IR.
In addition, our study did not show any differences in mitochondrial content measured as mtDNA copy number between lean and obese subjects with PCOS and controls. Decreased mitochondrial function due to decreased mitochondrial content has been demonstrated in young, insulin resistant first degree relatives of patients with type 2 diabetes (17), and patients with type 2 diabetes have consistently been shown to have lower muscle mitochondrial content than age- and BMImatched control subjects $(13,15,18)$. Whether decreases in mitochondrial content in subjects with type 2 diabetes is a cause or a consequence of the diabetic state remains an area of debate $(19,20)$. The discovery that PCOS was associated with decreased expression of genes involved in OXPHOS led to the hypothesis that aberrations in mitochondrial function could be the pathological feature of IR linking PCOS to an increased risk of development of diabetes (5). Certainly, an imbalance between substrate supply and oxidation in skeletal muscle could progressively lead to accumulation of lipid and lipid intermediates that inhibit insulin signaling (21). We are, however, not able to confirm this hypothesis as we did not find mitochondrial content or function to be reduced in

\section{$M$ value versus $\mathrm{GM} 3$}
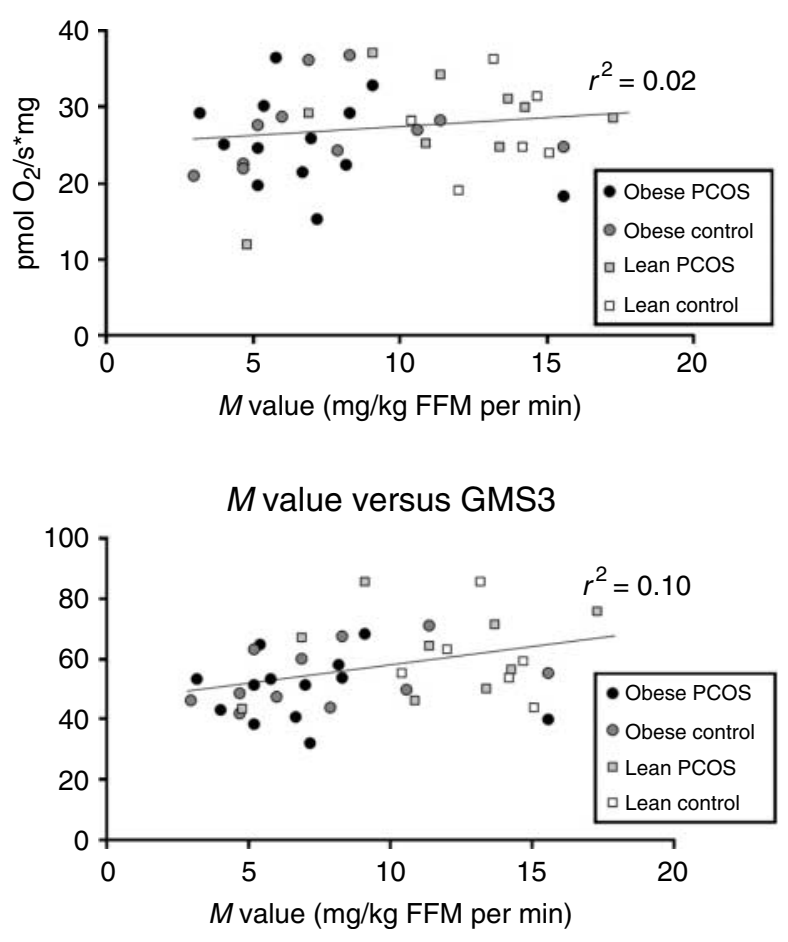

Figure 5 Correlation between mitochondrial respiration and insulin sensitivity measured during hyperinsulinemic-euglycemic clamping. No significant correlation could be found between respiratory state GM3 and the $M$ value or between respiratory state GMS3 and the $M$ value. $r^{2}$ values for the whole group of subjects are displayed in the graphs. Significant correlations between these parameters were not found in any of the subgroups. 
skeletal muscle of women with PCOS, but future studies should provide measurements of intramuscular lipid intermediates, especially diacylglycerol, to gain more information on this hypothesis.

Even though the obese PCOS had higher fasting insulin than obese controls and lower levels of complex I and uncoupled respiration (Fig. 4), IR does not appear to be the primary factor in altered mitochondrial function in PCOS on the basis of the overall findings of our study. This is in contrast to previous reports linking reduced OXPHOS gene expression in skeletal muscle to IR and PCOS (5), but it underscores the importance of carrying out detailed investigations of composite mitochondrial function (respirometry, content and enzyme activities) before inferring causality regarding mitochondrial function. The regulation of mitochondrial function is highly complex and takes place on many levels a part from gene expression.

Obesity is associated with excess electron transport reflected in higher respiratory capacity during uncoupling of respiration with FCCP. When mitochondria are uncoupled electrons can pass freely over the inner mitochondrial membrane, and electron transport and respiration is not limited by the negative feedback of increases in membrane potential and not limited by the activity of the ATP synthase. Electron transport is only limited by the ability of the mitochondria to transport electrons through the complexes of the respiratory chain, and this capacity to transport electrons seems to be larger in obese control subjects than obese subjects with PCOS. An ability to adapt to increased substrate flow to the muscle by increasing oxidation could potentially protect muscle from accumulation of lipid and IR. It has been demonstrated that overweight subjects with maintained insulin sensitivity exhibit increased lipid oxidation (22), so the fact that our group of obese PCOS subjects did not have increased excess electron transport capacity, could potentially increase their risk for intramuscular lipid accumulation and IR. The physiological significance of excess electron transport capacity is, however, far from understood (9).

In conclusion, the results of this demonstrate, using high-resolution respirometry that skeletal muscle mitochondrial respiratory capacity is not reduced in PCOS. Furthermore, mitochondrial content did not differ between our control and PCOS groups. These findings are in contrast to previous reports of reduced expression of OXPHOS genes in women with PCOS, and do not support a causal relationship between reduced mitochondrial function and skeletal muscle IR in PCOS.

\section{Declaration of interest}

The authors declare that there is no conflict of interest that could be perceived as prejudicing the impartiality of the research reported.

\section{Funding}

The authors thank the Danish Hospital Foundation for Medical Research, Region of Copenhagen, The Faroe Islands and Greenland; The Danish Diabetes Association; The Aage Bangs Foundation; Aase and Einar Danielsens Foundation; The Novo Nordisk Foundation; The Lundbeck Foundation; The Foundation of 1870 and Jacob Madsen and Olga Madsen's Foundation for financial support.

\section{Author contribution statement}

All authors were involved in data collection and analysis. All authors except for R Rabøl and P Schjerling contributed to project planning and all authors, except for M Skovbro were involved in manuscript preparation.

\section{Acknowledgements}

A special thanks to Susanne Reimar and Regitze Kraunsøe for expert technical assistance.

\section{References}

1 Ehrmann DA. Polycystic ovary syndrome. New England Journal of Medicine 2005352 1223-1236. (doi:10.1056/NEJMra041536)

2 Azziz R, Woods KS, Reyna R, Key TJ, Knochenhauer ES \& Yildiz BO. The prevalence and features of the polycystic ovary syndrome in an unselected population. Journal of Clinical Endocrinology and Metabolism 200489 2745-2749. (doi:10.1210/jc.2003032046)

3 Dunaif A. Insulin resistance and the polycystic ovary syndrome: mechanism and implications for pathogenesis. Endocrine Reviews 199718 774-800. (doi:10.1210/er.18.6.774)

4 Petersen KF \& Shulman GI. Etiology of insulin resistance. American Journal of Medicine 2006119 S10-S16. (doi:10.1016/j.amjmed. 2006.01.009)

5 Skov V, Glintborg D, Knudsen S, Jensen T, Kruse TA, Tan Q, Brusgaard K, Beck-Nielsen H \& Hojlund K. Reduced expression of nuclear-encoded genes involved in mitochondrial oxidative metabolism in skeletal muscle of insulin-resistant women with polycystic ovary syndrome. Diabetes $2007 \quad 56$ 2349-2355. (doi:10.2337/db07-0275)

6 Mootha VK, Lindgren CM, Eriksson KF, Subramanian A, Sihag S, Lehar J, Puigserver P, Carlsson E, Ridderstrale M, Laurila E, Houstis N, Daly MJ, Patterson N, Mesirov JP, Golub TR, Tamayo P, Spiegelman B, Lander ES, Hirschhorn JN, Altshuler D \& Groop LC. PGC-1alpha-responsive genes involved in oxidative phosphorylation are coordinately downregulated in human diabetes. Nature Genetics 200334 267-273. (doi:10. 1038/ng1180)

7 Patti ME, Butte AJ, Crunkhorn S, Cusi K, Berria R, Kashyap S, Miyazaki Y, Kohane I, Costello M, Saccone R, Landaker EJ, Goldfine AB, Mun E, DeFronzo R, Finlayson J, Kahn CR \& Mandarino LJ. Coordinated reduction of genes of oxidative metabolism in humans with insulin resistance and diabetes: potential role of PGC1 and NRF1. PNAS 2003100 8466-8471. (doi:10.1073/pnas.1032913100)

8 Victor VM, Rocha M, Banuls C, Sanchez-Serrano M, Sola E, Gomez M \& Hernandez-Mijares A. Mitochondrial complex I impairment in leukocytes from polycystic ovary syndrome patients with insulin resistance. Journal of Clinical Endocrinology and Metabolism 200994 3505-3512. (doi:10.1210/jc.20090466)

9 Gnaiger E. Capacity of oxidative phosphorylation in human skeletal muscle: new perspectives of mitochondrial physiology. International Journal of Biochemistry \& Cell Biology 200941 1837-1845. (doi:10.1016/j.biocel.2009.03.013) 
10 Svendsen PF, Nilas L, Norgaard K, Jensen JE \& Madsbad S. Obesity, body composition and metabolic disturbances in polycystic ovary syndrome. Human Reproduction 200823 2113-2121. (doi:10. 1093/humrep/den211)

11 Svendsen PF, Madsbad S \& Nilas L. The insulin-resistant phenotype of polycystic ovary syndrome. Fertility and Sterility 201094 1052-1058. (doi:10.1016/j.fertnstert.2009.04.008)

12 Rabol R, Svendsen PF, Skovbro M, Boushel R, Haugaard SB, Schjerling P, Schrauwen P, Hesselink MK, Nilas L, Madsbad S \& Dela F. Reduced skeletal muscle mitochondrial respiration and improved glucose metabolism in nondiabetic obese women during a very low calorie dietary intervention leading to rapid weight loss. Metabolism 200958 1145-1152. (doi:10.1016/j.metabol.2009. 03.014)

13 Boushel R, Gnaiger E, Schjerling P, Skovbro M, Kraunsoe R \& Dela F. Patients with type 2 diabetes have normal mitochondrial function in skeletal muscle. Diabetologia $2007 \mathbf{5 0} 790-796$. (doi:10.1007/s00125-007-0594-3)

14 Larsen S, Ara I, Rabol R, Andersen JL, Boushel R, Dela F \& Helge JW. Are substrate use during exercise and mitochondrial respiratory capacity decreased in arm and leg muscle in type 2 diabetes? Diabetologia 200952 1400-1408. (doi:10.1007/ s00125-009-1353-4)

15 Rabol R, Hojberg PM, Almdal T, Boushel R, Haugaard SB, Madsbad S \& Dela F. Effect of hyperglycemia on mitochondrial respiration in type 2 diabetes. Journal of Clinical Endocrinology and Metabolism 200994 1372-1378. (doi:10.1210/jc.20081475)

16 Rabol R, Boushel R, Almdal T, Hansen CN, Ploug T, Haugaard SB, Prats C, Madsbad S \& Dela F. Opposite effects of pioglitazone and rosiglitazone on mitochondrial respiration in skeletal muscle of patients with type 2 diabetes. Diabetes, Obesity E Metabolism 201012 806-814. (doi:10.1111/j.1463-1326. 2010.01237.x)

17 Morino K, Petersen KF, Dufour S, Befroy D, Frattini J, Shatzkes N, Neschen S, White MF, Bilz S, Sono S, Pypaert M \& Shulman GI. Reduced mitochondrial density and increased IRS-1 serine phosphorylation in muscle of insulin-resistant offspring of type 2 diabetic parents. Journal of Clinical Investigation $20051153587-$ 3593. (doi:10.1172/JCI25151)

18 Kelley DE, He J, Menshikova EV \& Ritov VB. Dysfunction of mitochondria in human skeletal muscle in type 2 diabetes. Diabetes $2002512944-2950$. (doi:10.2337/diabetes.51.10.2944)

19 Holloszy JO. Skeletal muscle "mitochondrial deficiency" does not mediate insulin resistance. American Journal of Clinical Nutrition 200989 463S-466S. (doi:10.3945/ajcn.2008.26717C)

20 Rabol R, Boushel R \& Dela F. Mitochondrial oxidative function and type 2 diabetes. Applied Physiology, Nutrition, and Metabolism 2006 31 675-683. (doi:10.1139/h06-071)

21 Shulman GI. Cellular mechanisms of insulin resistance. Journal of Clinical Investigation 2000106 171-176. (doi:10.1172/JCI10583)

22 Perseghin G, Scifo P, Danna M, Battezzati A, Benedini S, Meneghini E, Del Maschio A \& Luzi L. Normal insulin sensitivity and IMCL content in overweight humans are associated with higher fasting lipid oxidation. American Journal of Physiology. Endocrinology and Metabolism 2002283 E556-E564. (doi:10. 1152/ajpendo.00127.2002)

Received 10 May 2011

Revised version received 19 June 2011

Accepted 28 July 2011 